CASE REPORT

\title{
The value of c-kit mutational analysis in a cytokeratin positive gastrointestinal stromal tumour
}

\author{
G Rossi, G Sartori, R Valli, F Bertolini, N Bigiani, L Schirosi, A Cavazza, G Luppi
}

J Clin Pathol 2005;58:991-993. doi: 10.1136/icp.2004.024364

The expression of cytokeratins in gastrointestinal stromal tumours (GISTs) is rare and may lead to diagnostic confusion when it occurs. This report describes a metastatic GIST that stained strongly for cytokeratins, CD117, and CD34 in a patient who was previously diagnosed with gastric epithelioid angiosarcoma. A review of both tumours showed the same histological and immunohistochemical profiles, and c-kit molecular analysis revealed an insertional mutation at codon 558 of exon 11 in both tumours. Thus, pathologists should be aware that GISTs can occasionally express cytokeratins, and that c-kit mutational investigations may have a key diagnostic role and may prevent diagnostic mistakes that could have important clinical implications.

G astrointestinal stromal tumours (GISTs) are the most common mesenchymal neoplasms of the digestive tract. ${ }^{1}$ The identification of KIT (CDI17) as a specific immunohistochemical marker, ${ }^{2}{ }^{3}$ together with the discovery of gain of function mutations in the protooncogene c-kit in most GISTs ${ }^{4-6}$ represent crucial steps in the definition of this tumour as a biologically distinctive malignancy and, most importantly, in the development of an alternative therapeutic approach using targeted treatment with a specific KIT inhibitor, imatinib mesylate (formerly known as STI57l, Glivec $\left.^{\circledR}\right){ }^{7}$

\section{"Gastrointestinal stromal tumours are the most common mesenchymal neoplasms of the digestive tract"}

In routine practice, when faced with a soft tissue tumour of the gastrointestinal tract, surgical pathologists usually use a small immunohistochemical panel of antibodies to support the morphological picture, including CD117, CD34, smooth muscle actin, desmin, and S-100 protein. ${ }^{8}$ In fact, almost all GISTs are CD117 positive and about $60-70 \%$ are CD34 immunopositive, whereas the other markers are usually negative. ${ }^{8}$ In this context, immunostaining for cytokeratins may be helpful to rule out the possibility of a sarcomatoid carcinoma, because GISTs are usually negative for epithelial markers.

Here, we describe a gastric GIST initially diagnosed as epithelioid angiosarcoma which metastasised to the pelvis and strongly expressed cytokeratins and CDI17. Molecular analysis revealed the presence of a c-kit mutation in exon 11 in both tumours, thus confirming the diagnosis of GIST. The exceptionally rare occurrence of cytokeratin immunostaining in GISTs is also discussed.

\section{CASE REPORT}

In July 2003, a 32 year old woman originating from Ghana was admitted to our hospital as a result of a lack of appetite and abdominal discomfort resulting from a painful mass in the right iliac fossa. Her medical history revealed that four years previously she had undergone total gastrectomy plus omentectomy at another hospital for a gastric malignancy diagnosed as epithelioid angiosarcoma, for which she received adjuvant chemotherapy (doxorubicin and ifosfamide). Based on her previous diagnosis, metastatic disease was clinically suspected. Abdominal ultrasonography and a computerised tomography scan confirmed the presence of a large $(8 \mathrm{~cm}$ across $)$ solid mass in the right iliac region and a partially cystic mass $(6 \mathrm{~cm})$ in the left one. Radiological investigations revealed no significant signs in the other abdominal organs or regional lymph nodes. A chest $x$ ray was unremarkable. Tumour markers (CA125 and carcinoembryonic antigen) and laboratory studies were negative. The patient then underwent complete surgical excision of the pelvic masses and right adnexectomy. The surgical specimen, routinely formalin fixed and paraffin wax embedded, grossly showed a grey/whitish cut surface with bloody areas. At histology, the tumour appeared as a proliferation of polygonal to round epithelioid cells, with abundant lightly eosinophilic cytoplasm and centrally located nucleus (fig lA). Clear perinuclear vacuoles were seen. Mitotic activity was very high ( 18 mitoses $/ \times 50$ high power field) and foci of punctate necrosis were found.

Immunohistochemistry revealed that the neoplastic elements were strongly positive for CD117 (polyclonal antibody, A4502; 1/200 dilution; no antigen retrieval; Dako, Glostrup, Denmark) (fig 1B), CD34 (monoclonal QB-END/10; 1/40 dilution; antigen retrieval, microwave; Novocastra, Newcastle upon Tyne, UK) bcl-2 (monoclonal; 1/10 dilution; antigen retrieval, microwave; Ventana, Tucson, Arizona, USA), AEl/ AE3 (monoclonal; prediluted; antigen retrieval, protease; Ventana), MNF116 (monoclonal; 1/1500 dilution; antigen retrieval, protease; Dako), CAM5.2 (monoclonal; 1/50 dilution; no antigen retrieval; Becton Dickinson, San Josè, California, USA) (fig 1C), but did not stain for high molecular weight cytokeratins (monoclonal 34ßE12; 1/500 dilution; antigen retrieval, microwave; Dako), CD31 (monoclonal JC/ 70A; prediluted; antigen retrieval, protease; Ventana), epithelial membrane antigen (monoclonal E29; 1/300 dilution; no antigen retrieval; Dako), desmin (monoclonal D33; 1/10 dilution; antigen retrieval, microwave; Dako), smooth muscle actin (monoclonal 1A4; 1/20 dilution; no antigen retrieval; Biogenex, San Ramon, California, USA), S-100 (polyclonal; 1/5 dilution; no antigen retrieval; NeoMarkers, Freemont, California, USA), chromogranin (monoclonal DAK-A3; 1/100 dilution; antigen retrieval, microwave; Dako), HMB-45 (monoclonal HMB-45; prediluted; antigen retrieval, microwave; Ventana), or h-caldesmon (monoclonal hCD; 1/100 dilution; antigen retrieval, microwave; Dako).

Abbreviations: GIST, gastrointestinal stromal tumour; PCR, polymerase chain reaction 

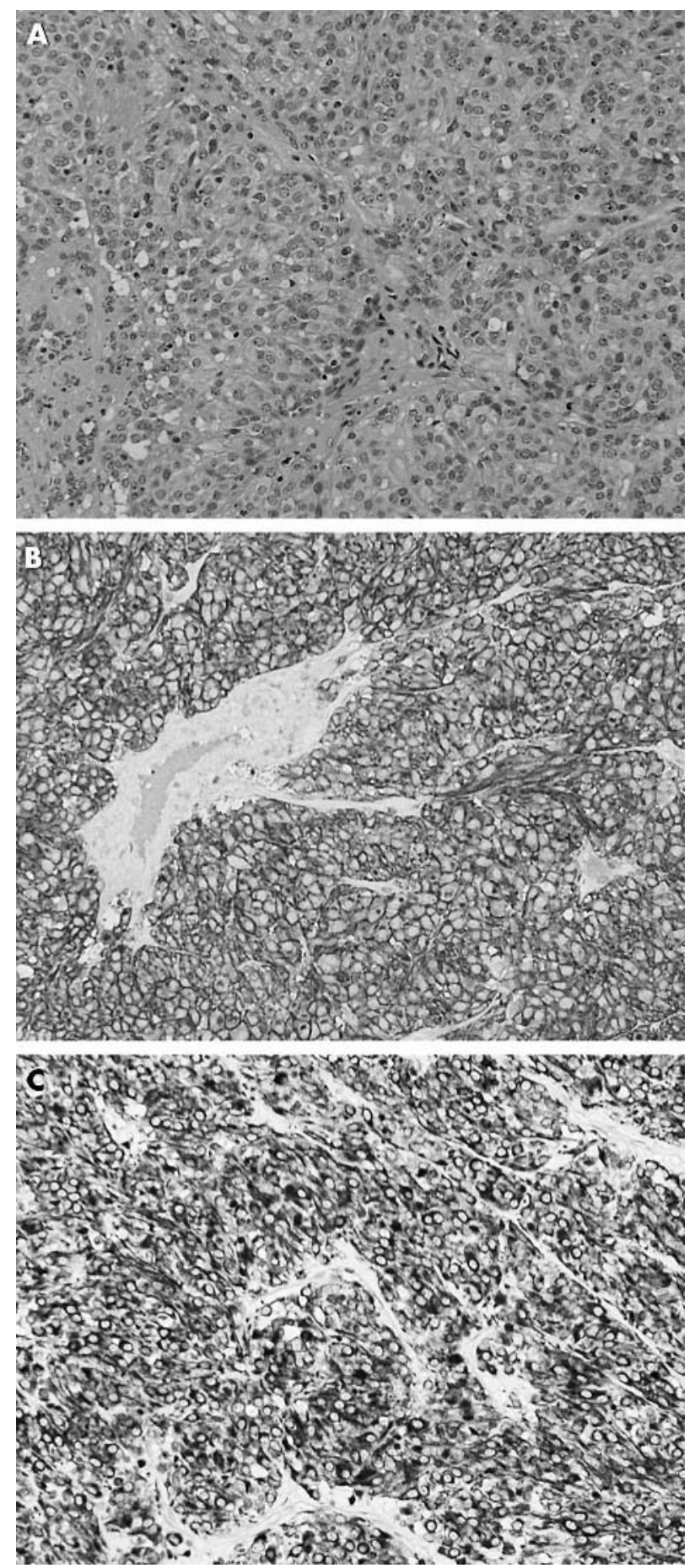

Figure 1 (A) The tumour appeared as an epithelioid cell proliferation consistent with gastrointestinal stromal tumour and was strongly immunopositive for (B) CD1 17 and (C) cytokeratin.

Several $5 \mu \mathrm{m}$ thick sections obtained from a representative paraffin wax embedded block were dewaxed by xylene and tumour DNA was extracted using a laser capture microdissection method (LaserScissor-PRO300; Olympus, Tokyo, Japan). Microdissected tumour cells were subjected to proteinase $\mathrm{K}$ treatment in extraction buffer $(50 \mathrm{mM}$ Tris/HCl (pH 8.0), ImM EDTA, 0.5\% Tween 20) and then incubated overnight at $37^{\circ} \mathrm{C}$. Polymerase chain reaction (PCR) was performed in $10 \mu \mathrm{l}$ reactions containing $1.0 \mu \mathrm{l}$ DNA, $10 \mathrm{mM}$ Tris/HCl (pH 8.3), 40mM KCl, $1.0-1.5 \mathrm{mM} \mathrm{MgCl}_{2}, 200 \mathrm{mM}$ each dNTP, 20pM of each primer, and 0.25 U Platinum Taq polymerase. The PCR reaction was carried out on a Uno II Thermoblock (Biometra, Gottingen, Germany). Initial denaturation at $94^{\circ} \mathrm{C}$ for three minutes was followed by 41 cycles of denaturation at $95^{\circ} \mathrm{C}$ for one minute, annealing at $53-58^{\circ} \mathrm{C}$ for 40 seconds, and extension at $72^{\circ} \mathrm{C}$ for 35 seconds, with a final extension step of five minutes at $72^{\circ} \mathrm{C}$. The amplified DNA was eletrophoresed on a $1 \%$ low melt agarose gel for one hour. The amplification products were then excised from the gel and purified using Wizard PCR Preps-DNA purification system (Promega Corp, Madison, Wisconsin, USA), as indicated by the manufacturer. The PCR products were then sequenced in both directions with the BigDye Terminator (Applied Biosystems, Weiterstadt, Germany) sequencing kit, using the same primers as those used for PCR. PCR products were finally purified by Centri-Sep spin columns and subsequently analysed using the ABIPrism 310 sequence analyser (Applied Biosystems). The following oligonucleotide primers were used to amplify c-kit exons 9 and 11: exon 9 (forward, 5' -ATG CTC TGC TTC TGT ACT GCC-3'; reverse, 5' CAG AGC CTA AAC ATC CCC TTA-3'; 238 bp; $58^{\circ} \mathrm{C}$ annealing temperature), exon 11 (forward, 5'-CTA TTT TTC CCT TTC TCC CC-3'; reverse, 5'-TAC CCA AAA AGG TGA CAT GG-3'; $193 \mathrm{bp} ; 53^{\circ} \mathrm{C}$ annealing temperature).

Molecular analysis revealed a c-kit mutation consisting of an insertion of the TCC nucleotides at codon 558 (fig 2). The histological review of the previously diagnosed gastric tumour showed a similar epithelioid malignancy with immunohistochemical features identical to those seen in the pelvic metastatic deposits. Most importantly, the original gastric tumour revealed the same insertion mutation found in the metastatic deposits. Thus, a diagnosis of metastatic GIST was rendered and the patient started treatment with Glivec ${ }^{\circledR}$ (400 mg/day). She is alive and well at 22 months follow up.

\section{DISCUSSION}

GISTs are well defined clinicopathological tumours and are the most frequent mesenchymal malignancies found in the digestive tract. ${ }^{19}$ They are characterised by the expression of the type III tyrosine kinase KIT, the product of the protooncogene c-kit, recognised by the antibody CD117. ${ }^{1-68} 8$ Most importantly, c-kit activating gain of function mutations occur in most GISTs, whereas a small subset of GISTs are KIT negative and lack c-kit mutations. ${ }^{9}$ These KIT negative tumours show activating mutations of the PDGFRA gene, encoding the type III tyrosine kinase PDGFR- $\alpha .^{10}$

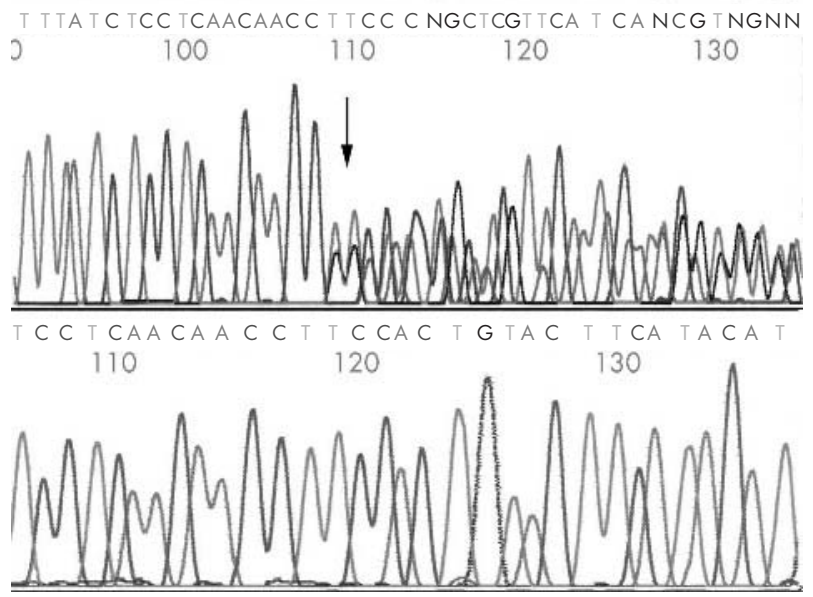

Figure 2 Molecular analysis revealed the presence of an insertion mutation on codon 558 of the c-kit exon 11 (arrow). 


\section{Take home messages}

- We describe a metastatic gastrointestinal stromal tumour (GIST) that stained strongly for cytokeratins, CD1 17, and CD34 in a patient previously diagnosed with gastric epithelioid angiosarcoma

- Both tumours showed the same histological and immunohistochemical profiles, and c-kit molecular analysis revealed the same mutation in both tumours

- Thus, pathologists should be aware that GISTs can occasionally express cytokeratins and that c-kit mutational analysis may help in diagnosis and prevent mistakes that could have important clinical implications

Most GISTs display a spindle, epithelioid, or mixed (spindle/epithelioid) cell morphology, but they may show a broad spectrum of histological growth patterns that make the differential diagnosis difficult, and may be confused with smooth muscle tumours, desmoids, neural tumours, neuroendocrine tumours, inflammatory myofibroblastic tumour and fibroid polyp, synovial sarcoma, sarcomatoid carcinoma, and angiosarcoma. ${ }^{11}$ For this reason, immunostaining is mandatory, not only to achieve the diagnosis, but also because of the clinical implications of a diagnosis of GIST. As suggested by Fletcher et al, ${ }^{8}$ the diagnosis of GIST should be based not only on morphological grounds, but staining with a small panel of antibodies should also be performed to rule out other mimicking tumours. Here, we report a rare case of metastatic GIST strongly expressing cytokeratins, in addition to CD34 and CD117, and originally thought to be a gastric epithelioid angiosarcoma.

Nga and colleagues ${ }^{12}$ recently reported a case of cytokeratin positive pleural metastatic GIST initially suspected to be lung carcinoma and found two other GISTs showing focal, dot-like immunoreactivity for CAM5.2 in a series of 41 GISTs. Of note, all these cytokeratin positive GISTs had a gastric origin. Although extremely rare, this has been described previously by Miettinen et al, ${ }^{13}$ who found two keratin 18 positive tumours in a series of 57 anorectal GISTs. In both studies, no GIST showed immunostaining for other epithelial markers (such as AEl/AE3 and epithelial membrane antigen).

"Insertional mutation in codon 558 of c-kit exon 11 has been reported previously in gastrointestinal stromal tumours, and seems to be related to a good clinical response rate with $\mathrm{STI} 571^{\prime \prime}$

Apart from GIST, CD117 expression has been reported in several other tumours, mainly as a result of the presence of different commercially available antibodies against KIT and the use of inappropriate technical protocols. ${ }^{14}$ Among tumours coexpressing CD34 and cytokeratins, CD117 immunoreactivity has been reported in angiosarcoma only. ${ }^{15}$ Even though angiosarcomas may primarily arise from the stomach, they lack c-kit mutations and generally carry a worse prognosis than that of GIST. ${ }^{15}$

Mutational analysis of c-kit appears to be extremely helpful, if not mandatory, in confirming the diagnosis of GIST when this tumour shows an unusual presentation on clinical, morphological, and/or immunohistochemical grounds, as in our case. In addition, insertional mutation in codon 558 of c-kit exon 11 has been reported previously in GIST, and seems to be related to a good clinical response rate with STI571. ${ }^{6} 16$

Finally, pathologists should be aware that, although unusual, GISTs may express cytokeratins, and this should be kept in mind when dealing with small biopsies to prevent an erroneous diagnosis.

\section{Authors' affiliations}

G Rossi, G Sartori, R Valli, N Bigiani, L Schirosi, Integrated Department of Diagnostic and Laboratory Services and Legal Medicine, Section of Pathologic Anatomy, University of Modena and Reggio Emilia, via del Pozzo 71, 41100 Modena, Italy

F Bertolini, G Luppi, Department of Oncology and Haematology, Section of Oncology, University of Modena and Reggio Emilia

A Cavazza, Department of Pathology, S. Maria Nuova Hospital, Viale Risorgimento 80, 42100 Reggio Emilia, Italy

The patient gave her full permission for this case report to be published

Correspondence to: $\operatorname{Dr} G$ Rossi, Dipartimento Integrato di Servizi Diagnostici, di Laboratorio e di Medicina Legale, Sezione di Anatomia Patologica, Università di Modena e Reggio Emilia, via del Pozzo 71, 41100 Modena, Italy; rossi.giulio@unimo.it

Accepted for publication 17 January 2005

\section{REFERENCES}

1 Miettinen M Lasota J. Gastrointestinal stromal tumors-definition, clinical, histological, immunohistochemical, and molecular genetic features and differential diagnosis. Virchows Arch 2001;438:1-12.

2 Kindblom LG, Remotti HE, Aldenborg F, et al. Gastrointestinal pacemaker cell tumor (GIPACT). Gastrointestinal stromal tumors show phenotypic characteristics of the interstitial cells of Cajal. Am J Pathol 1998; 152:1259-69

3 Sarlomo-Rikala M, Kovatich AJ, Barusevicius A, et al. CD1 17: a sensitive marker for gastrointestinal stromal tumors that is more specific than CD34. Mod Pathol 1998;1 1:728-34.

4 Hirota S, Isozaki K, Moriyama Y, et al. Gain-of-function mutations of c-kit in human gastrointestinal stromal tumors. Science 1998;279:577-80.

5 Rubin BP, Singer S, Tsao C, et al. KIT activation is a ubiquitous feature of gastrointestinal stromal tumors. Cancer Res 2001;61:8118-21.

6 Heinrich MC, Corless CL, Demetri GD, et al. Kinase mutations and imatinib response in patients with metastatic gastrointestinal stromal tumor. J Clin Oncol 2003;21:4342-9.

7 Joensuu H, Roberts PJ, Sarlomo-Rikala M, et al. Effect of the tyrosine kinase inhibitor STI571 in a patient with metastatic gastrointestinal stromal tumor. N Engl J Med 2001;344:1052-6.

8 Fletcher CDM, Berman JJ, Corless C, et al. Diagnosis of gastrointestinal stromal tumors: a consensus approach. Hum Pathol 2002;33:459-65.

9 Corless C, Fletcher JA, Heinrich MC. Biology of gastrointestinal stromal tumors. J Clin Oncol 2004;22:3813-25.

10 Heinrich MC, Corless C, Duensing A, et al. PDGFRA activating mutations in gastrointestinal stromal tumors. Science 2003;299:708-10.

11 Suster S. Gastrointestinal stromal tumors. Semin Diagn Pathol 1996; 13:297-313.

12 Nga ME, Wong ASC, Wee A, et al. Cytokeratin expression in gastrointestinal stromal tumours: a word of caution. Histopathology 2002;40:480-1.

13 Miettinen M, Furlong M, Sarlomo-Rikala $M$, et al. Gastrointestinal stromal tumors, intramural leiomyomas, and leiomyosarcomas in the rectum and anus. A clinicopathologic, immunohistochemical, and molecular genetic study of 144 cases. Am J Surg Pathol 2001;25:1121-33.

14 Hornick JL, Fletcher CDM. Immunohistochemical staining for KIT (CD117) in soft tissue sarcomas is very limited in distribution. Am J Clin Pathol 2002; 117:188-93.

15 Miettinen M, Sarlomo-Rikala M, Lasota J. KIT expression in angiosarcomas and fetal endothelial cells: lack of mutations of exon 11 and exon 17 of c-kit. Mod Pathol 2000;13:536-41.

16 lijima S, Maesawa C, Sato N, et al. Gastrointestinal stromal tumour of the oesophagus: significance of immunohistochemical and genetic analyses of the c-kit gene. Eur J Gastroenterol Hepatol 2002;14:445-8. 